ÉGYPTE monde arabe

\section{Égypte/Monde arabe}

30-31 | 1997

Les visions de l'Occident dans le monde arabe

\title{
L'image de l'Occident chez les prêcheurs musulmans et coptes au Caire, aujourd'hui
}

Saadia Radi

\section{OpenEdition}

1 Journals

Édition électronique

URL : https://journals.openedition.org/ema/1611

DOI : 10.4000/ema.1611

ISSN : 2090-7273

Éditeur

CEDEJ - Centre d'études et de documentation économiques juridiques et sociales

Édition imprimée

Date de publication : 30 septembre 1997

Pagination : 159-171

ISSN : 1110-5097

\section{Référence électronique}

Saadia Radi, «L'image de l'Occident chez les prêcheurs musulmans et coptes au Caire, aujourd'hui », Égypte/Monde arabe [En ligne], 30-31 | 1997, mis en ligne le 08 juillet 2008, consulté le 07 juillet 2022. URL : http://journals.openedition.org/ema/1611 ; DOI : https://doi.org/10.4000/ema.1611

Ce document a été généré automatiquement le 7 juillet 2022.

Tous droits réservés 


\title{
L'image de l'Occident chez les prêcheurs musulmans et coptes au Caire, aujourd'hui
}

\author{
Saadia Radi
}

« Il est possible dira-t-on, sur le plan d'une

logique abstraite, que chaque culture soit incapable de porter un jugement vrai sur une autre, puisqu'une culture ne peut s'évader d'elle-

même et que son appréciation reste, par conséquent, prisonnière d'un relativisme sans

appel. » Claude Lévi-Strauss, Anthropologie structurale I.

1 Vouloir définir l'autre, vouloir préciser ses caractéristiques est peut être une façon détournée de se définir soi-même. Définir l'autre, c'est lui donner une identité et, par là même, se donner une identité ou se procurer les caractéristiques que l'on croit intrinsèques à sa propre identité. Il me semble que le discours que l'on tient sur sa propre identité change selon les situations. Ainsi, lorsqu'on parle de soi sans faire référence à d'autres, on parle de ses qualités, de ses défauts et de ce que l'on croit être ses spécificités, mais lorsqu'on parle de soi en se comparant à d'autres, on essaie d'abord de définir l'autre ou de définir ses caractéristiques, afin de se définir d'une façon implicite. Autrement dit, on parle de l'autre pour dire : lui a des qualités qui ne sont pas les miennes, donc il est différent de moi. Cette différence ou cette altérité, selon les propos des acteurs, constitue l'identité.

2 En traitant de l'identité, je pars donc du principe qu'elle n'est rien en soi ; elle est ce qu'en font les acteurs; elle est un signifiant flottant et mutable. Mais, bien qu'elle ne soit rien en soi, elle veut dire beaucoup pour celui qui s'y réfère et elle contient tout ce que l'on y met. Elle est vide de sens mais remplie de significations. Vide de sens, parce qu'elle ne contient précisément que ce que l'on y met, et remplie de significations, parce qu'elle permet de dire ce qu'on est ou ce qu'on croit être. De ce fait, elle est pour 
les acteurs sociaux et pour une certaine anthropologie, une substance objective et matérielle et c'est cette substantialité imaginée, à laquelle on croit fortement, qui nous donne le droit de dire que chaque culture possède une "spécificité spécifique ", que nous sommes différents des autres et que les autres sont différents de nous. On peut dire cela à propos des pays lointains, des pays proches et même d'un groupe donné à l'intérieur d'un même pays. Ce raisonnement peut être poussé à l'extrême, en ce qui concerne d'autres peuples, jusqu'à dire que l'on ne partage avec eux que le fait d'être tous des êtres humains. Lorsqu'on évoque cette différence identitaire cela implique d'une manière implicite ou explicite qu'on prétend posséder une culture typique et différente et que les autres ont aussi une culture typique et différente. La définition que les acteurs sociaux peuvent donner d'eux-mêmes n'est pas sans rappeler, dans une certaine mesure, les positions de Margaret Mead et du courant culturaliste.

Comme on l'a dit plus haut, l'identité n'est rien en soi mais elle est ce qu'on y met. Autrement dit, c'est un ensemble d'éléments construits et admis afin de se définir ou de définir l'autre. De ce fait, il est plus judicieux de remplacer le terme identité par « construit identitaire » (Ireton, à paraître). Ce construit identitaire peut paraître solide et immuable parce que, souvent, les personnes se définissent ou définissent l'autre d'une manière qui ne laisse pas subsister de doute sur ce qu'ils sont ou sur ce qu'ils croient être, comme lorsqu'ils disent, par exemple : « Nous sommes des gens qui avons telles et telles caractéristiques, nous agissons d'une manière bien précise, tout comme nos ancêtres ont pu le faire. » On parle de soi comme si on savait avec précision ce qu'on est ou ce que les autres sont, et comme si les choses sont dans la réalité exactement comme on les décrit. Mais cette construction de la réalité qui semble stable et inchangeable est en fait d'une grande fragilité. Des acteurs sociaux peuvent croire (du moins, c'est l'impression qu'ils donnent en parlant de ce qu'ils sont) dur comme fer, si j'ose dire, que leur identité est basée et construite sur un élément capital qui existe depuis la nuit des temps et qui continuera d'exister après eux. Mais cet élément fondateur peut pourtant être remplacé par un autre qui devient, à son tour, l'élément capital et la clé de ce que l'on nomme identité.

Mon but n'est pas, dans le texte qui suit, de décrire comment les prêcheurs cairotes se définissent ni comment ils définissent leur identité, mais quelle perception ils ont de l'Occident et des Occidentaux. Cependant, il est maintenant évident qu'en parlant des caractéristiques des autres, on est forcément amené à se comparer à eux et donc à se définir soi-même.

\section{Les prêcheurs musulmans du Caire}

5 Certes, nous sommes dans une époque où l'on ne peut plus affirmer l'existence d'une altérité totale entre l'Orient et l'Occident ni même d'une culture purement orientale et d'une autre purement occidentale. Les rues du Caire et le comportement des Cairotes ne sont pas réellement exotiques par rapport à ce qui se passe dans cette partie du monde que l'on nomme Occident et qui est présent en Orient par ses produits, son architecture, ses techniques. Comme l'écrivait déjà Lévi-Strauss (1973, p. 402) : «Ne voyions-nous pas le monde entier lui emprunter progressivement ses techniques, son genre de vie, ses distractions et jusqu'à ses vêtements ». Cet Occident omniprésent dans l'espace matériel, l'est aussi dans les discours. Malgré cela, il reste un imaginaire de Tailleurs. À part la corruption éthique que l'Occident est censé introduire en Orient, les 
prêcheurs égyptiens font abstraction de tout ce qu'il y a d'occidental dans la société égyptienne. Dans n'importe quel espace égyptien, qu'il soit modeste ou non, qu'il soit urbain ou rural, on trouve des produits «occidentaux ». Ces produits en eux-mêmes sont considérés comme des objets locaux et qui font partie intégrante de cet espace. On ne se pose de question ni sur leur provenance ni sur leur créateur. En revanche, on peut poser des questions sur leur fonction. La télévision, qui a une présence très marquée jusque dans les taxis, fait partie d'un ensemble d'objets « naturels » ou " normaux » que l'on possède. Cet objet, en tant que « matériel », n'a jamais donné lieu à polémique ; au contraire, ce sont les programmes que la télévision diffuse qui sont sujet à débat : ils sont stigmatisés comme immoraux et responsables de la corruption de l'éthique des Égyptiens. On peut taxer de corrupteur certains programmes égyptiens mais ce sont surtout les programmes «occidentaux » et turcs captés par le câble que l'on critique. Pour cette raison, on est allé jusqu'à interdire le câble dans certaines localités du nord de l'Égypte. Ce ne sont donc pas les objets «occidentaux» que l'on conteste - et pourquoi les contester à partir du moment où ils font partie du mode de vie endogène -, mais ce qu'on en fait. Autrement dit, la télévision et ce qu'elle transmet sont deux choses distinctes; l'objet est là, il fait partie intégrante de notre monde, mais les programmes sont autre chose, et surtout les programmes « occidentaux » qui ont pour «but manifeste» de corrompre la morale. Malgré cette présence de l'Occident en Égypte, à travers ses produits devenus endogènes, on continue de définir l'Occident comme un monde étrange et étranger. Autrement dit, les Cairotes adoptent une vision culturaliste où l'Égyptien se définit comme ayant des "spécificités spécifiques » et où l'autre est défini de la même manière.

6 Les " hommes de religion » (rigâl al-dîn) partagent aussi cette vision et en font part à leurs fidèles pendant les prêches ou par l'intermédiaire de cassettes enregistrées. Il faut rappeler que cette appellation est contestée par certains musulmans qui estiment qu'elle ne peut qualifier que le corps ecclésiastique chrétien: en effet, les prêcheurs musulmans ne sont pas un groupe ayant une formation religieuse ou des diplômes délivrés par l'université d'Al-Azhar. Ils peuvent avoir n'importe quelle formation, en médecine ou en biologie par exemple, ou n'avoir aucune formation. Cette question de la légitimité des prêcheurs est actuellement posée avec acuité sur la scène publique égyptienne, à cause d'une loi qui interdit à quiconque de prêcher sans l'autorisation du ministère des Affaires religieuses. La loi prévoit une peine de prison d'un mois et une amende qui varie entre cent et trois cents livres. Elle a provoqué ou au moins rendu public les conflits qui opposent, à Al-Azhar, les oulémas et une partie des prêcheurs au ministère en question. Certains oulémas d'Al-Azhar refusent l'application de cette loi aux diplômés de l'université en arguant que les Azharites n'ont pas besoin d'avoir une autre autorisation pour prêcher parce qu'ils ont déjà celle d'Al-Azhar (al-Siyâsî al-Masrî, 09/06/1996). D'autres oulémas refusent l'application de cette loi pour toute personne qui désire prêcher en soutenant que la $D a^{\prime \prime} w a$ islâmiyya n'a pas besoin de lois et que l'État ne doit pas intervenir dans la relation que le musulman entretient avec la mosquée (al-Siyâsî al-Masrî, 09/06/1996). On va même jusqu'à dire qu'il ne faut pas appliquer aux mosquées le même genre de lois qu'on applique aux bars, aux casinos ou cabarets et que, depuis les débuts de l'islam, n'importe quel musulman a le droit de prêcher en terre d'islam et même ailleurs (al-Sha'b, 12/06/1996). Le ministre des Waqfs justifie cette loi en disant qu'elle a pour but de protéger les gens des idées « extrémistes et déviantes » qui circulent actuellement au nom de l'islam et de les protéger aussi des 
idées fausses, de l'incitation au terrorisme et à la violence contre les musulmans et les non-musulmans (al-Akhbâr, 09/09/1996).

\section{La rhétorique de l'altérité avec l'Occident}

7 Pour le prêcheur musulman, l'Occident et les Occidentaux représentent l'altérité totale : «l'Orient est l'Orient et l'Occident est l'Occident » (al-gharb gharb wa al-charq charq). Ces deux truismes, cités chaque fois au début des entretiens que j'ai eus avec eux, signifient que les Orientaux ne partagent avec les Occidentaux que le fait d'être humains. Mais cette manière de vouloir distinguer l'autre et de se distinguer de lui n'est qu'une apparence. Cette altérité sans limite est en fait basée sur deux éléments fondamentaux.

8 Le premier élément qui justifie cette altérité est tout d'abord la religion: "Nous sommes des musulmans et eux, ce sont des chrétiens ou plutôt de mauvais chrétiens, parce qu'ils ont falsifié la Bible; et même le texte qu'ils ont actuellement, ils ne le respectent pas. »Cela veut dire que, si on n'est pas de la même religion, on ne peut se ressembler. Cette manière de penser est très explicite chez certains prêcheurs qui n'acceptaient pas que je leur demande: "Y a-t-il des différences entre l'Orient et l'Occident ? » et qui me conseillaient de reformuler ma question de la façon suivante : « Y a-t-il des différences entre les musulmans et les non-musulmans? » Le deuxième élément qui explique l'altérité et qui stigmatise l'autre occidental est «l'anarchie des mœurs » ou « la déviance sexuelle », généralement exprimée dans les termes suivants : «Les filles et les garçons ont des relations sexuelles en dehors du mariage; la femme peut en avoir avec une autre femme et l'homme avec un autre homme; la femme mariée peut avoir un ou plusieurs amants. "

Cette manière de voir l'Occident n'est pas spécifique aux prêcheurs; elle est partagée par la plupart des gens. On peut le constater en discutant avec un chauffeur de taxi, un boucher, un étudiant, un fonctionnaire, un médecin ou en feuilletant des quotidiens égyptiens. Ici, je dois avouer que mes entretiens avec des prêcheurs musulmans et des prêtres de l'église copte orthodoxe m'ont apporté peu d'informations. On vient de le voir, la distinction Occident/Orient repose sur deux distinctions sommaires seulement. Cela est sûrement dû à la nature du sujet lui-même : il n'y a pas plus pauvre que les stéréotypes et ces éléments sont des stéréotypes. Certes, les stéréotypes sont pauvres en quantité et riches de significations, tout au moins dans la pensée de ceux qui les utilisent. Mais, peut-on prendre ce discours pour la description de la réalité quotidienne? Lorsqu'on stigmatise l'Occident et l'Occidental, on évoque une catégorie abstraite, une construction détachée de cette réalité-là. Mais lorsque cette catégorie abstraite devient une réalité, c'est-à-dire, lorsque je côtoie un Occidental ou des Occidentaux, je ne les traite ni comme des individus qui ne respectent pas leur religion ni comme des individus qui n'ont pour but que d'avoir des relations sexuelles, mais je passe à un autre registre et je mets de côté mes stéréotypes ; je passe au registre de la réalité, celui de la proximité. N'est-on pas fier au Caire de pouvoir dire : «J'habite dans un immeuble très bien et d'ailleurs, la plupart de mes voisins sont des khawagât [terme qui désigne les étrangers non arabes et surtout les Européens]. " D'une manière caricaturale, cela me rappelle l'attitude chère à certains Français : "L'Arabe que je connais est très bien, il n'est pas comme les autres ", c'est-à-dire, en définitive : la proximité n'est jamais conforme aux stéréotypes. 
10 Selon Schütz (1987), le monde est livré à notre expérience et à notre interprétation, mais cette interprétation est basée sur une expérience acquise en même temps qu'héritée. Cette deuxième forme d'expériences qu'il appelle «connaissances disponibles » fonctionne comme schèmes de référence. Elles nous permettent de vivre dans un monde d'êtres et d'objets qui sont plus ou moins définis et dotés de caractéristiques stables. Ces objets ne sont pas perçus comme isolés ou dotés de caractéristiques spécifiques; ils prennent place à l'intérieur d'un univers familier. Ainsi, le monde extérieur est-il expérimenté sous formes d'ensembles - des «montagnes», des «arbres », d'« autres hommes » - qu'on ne peut apercevoir qu'en tant qu'entités dépouillées de toute caractéristique individuelle. Donc, dans ce registre, lorsqu'on parle de l'Occident ou des Occidentaux, on les classe ou on les cite dans la catégorie "autres hommes » et on leur attribue des caractéristiques qu'ils partagent tous. Autrement dit: tous les Occidentaux sont pareils, tous les Occidentaux fonctionnent de la même manière, tous les Occidentaux sont fondus dans le même moule. L'exemple du chien, donné par Schütz, illustre le deuxième volet de ma démonstration. On peut n'avoir jamais vu un setter irlandais mais, si on en voit un, on sait que c'est un animal et qu'il est un chien. Si on demande : "Il est de quelle race ce chien?", cette question fait apparaître la particularité de ce chien par rapport aux autres races de chien. Si je connais ce chien, ce sont ses caractéristiques personnelles qui, bien sûr, s'imposent à moi. Ainsi, lorsqu'on parle des Occidentaux en général, on les définit comme un groupe d'individus dotés de caractéristiques non individuelles. Mais, lorsqu'on sort de cette catégorie "abstraite » et lorsqu'on se retrouve dans des situations bien précises avec un Occidental ou des Occidentaux, on tient compte des caractéristiques individuelles et on laisse de côté les stéréotypes. Mais cela n'empêche pas de ressortir, dans d'autres situations, sa petite réserve de stéréotypes. En fait, les stéréotypes sont indépendants de l'expérience réelle de l'Occident et des Occidentaux.

11 Aux deux éléments stigmatisants dont j'ai parlé plus haut - religion et liberté sexuelle on peut en ajouter un autre qui leur est mêlé et qui est, à la fois, un objet de fascination et de rejet: c'est la «liberté» (hurriyya). Cette notion est ambivalente. La liberté occidentale est appréciée et même jalousée dans certains domaines comme ceux de la liberté d'expression et de la liberté politique. À ce propos, les prêcheurs musulmans disent :

«En Occident, les gens peuvent exprimer ce qu'ils veulent, ils peuvent critiquer ouvertement leur président, ils peuvent dire qu'ils sont pour ou contre une décision politique, ils peuvent manifester dans les rues pour montrer qu'ils ne sont pas contents de quelque chose.»

Mais, cette même notion est aussi diabolisée lorsqu'elle touche le domaine de l'éthique. Les prêcheurs musulmans disent alors: "La liberté a des limites, si l'on donne beaucoup de liberté à une fille, ce sera une fille perdue, si l'on donne trop de liberté à un garçon, il ne va pas connaître ses limites, il va peut-être consommer de l'alcool ou même des drogues. La liberté sans limites provoque la déviance. » Ou encore :

«La liberté, c'est avoir du contrôle sur soi, avoir une maîtrise de soi, ne pas être esclave de désirs bien précis. Un être qui est esclave de ses désirs n'est pas un être libre. Les Occidentaux ont mal compris la liberté, la preuve, c'est le grand nombre d'enfants illégitimes en Occident. Au nom de la liberté, les femmes ont des relations sexuelles avec un homme qui n'est pas leur mari ou même avec plusieurs hommes, elles ne savent même pas qui est le père de leur enfant, cet enfant risque de se marier avec sa sœur sans le savoir. Ces enfants sont victimes de la liberté qui est 
mal comprise par les parents. Notre religion est bien claire sur ce sujet, et elle ne permet pas ces choses-là. »

On constate ici que la question de la « liberté » fonctionne à l'intérieur de la religion et de l'éthique musulmane ou " orientale ». En d'autres termes, la liberté ne peut être que religieuse et morale. Lorsqu'elle dépasse une certaine limite, elle devient anarchie et dissolution. Selon les prêcheurs, on n'est véritablement libre que lorsqu'on est enchaîné (muqayyad). On est enchaîné par les prescriptions islamiques qu'il faut respecter, comme la prière, le jeûne, l'aumône, etc., et libre dans l'ijtihâd (effort d'interprétation personnelle du Coran), parce qu'on peut discuter de tout mais toujours dans le cadre de l'islam. Le terme enchaîné peut paraître péjoratif ou peut au moins signifier «contrainte». Mais les acteurs, lorsqu'ils évoquent le mot muqayyad, ne se situent absolument pas dans une rhétorique péjorative ni ne considèrent qu'ils évoquent quelque chose de contraignant comme le serait une contrainte physique. Le fait d'être enchaîné est un état intérieur, un état psychologique dont bénéficient les "personnes intègres", les "gens bien». Selon Abdallah Laroui (1986), le terme hurriyya, dans le Coran et le figh (jurisprudence islamique) exprime un état social, hurr (libre) s'opposant à 'abd (esclave); il s'applique aussi aux communautés ou institutions affranchies de toute imposition. Chez les mystiques - toujours selon Laroui - il exprime le processus qui consiste à se détacher du monde. Ce qui nous intéresse ici, ce ne sont ni les définitions du mot "liberté» dans les pays arabo-musulmans ni le fait de déterminer - comme prétend le faire Abdallah Laroui - s'il existe une liberté propre à la tradition musulmane. Ce qui nous intéresse, c'est la manière dont les prêcheurs la comprennent. Pour eux, elle a un lien intrinsèque avec la morale et avec la religion, et tout comportement immoral et irréligieux ne saurait donc pas ressortir de la «liberté bien comprise ». Cet argument semble relever de la philosophie morale classique selon laquelle l'homme enchaîné par ses passions n'est pas un homme libre. En effet, ce thème est constant chez les philosophes occidentaux du XVII ${ }^{e}$ et XVIII ${ }^{e}$ siècles. On peut penser qu'il s'agit là d'un héritage commun à la pensée occidentale et à la pensée arabe. Cependant, il est impossible de supposer que mes interlocuteurs font, même implicitement, référence à l'une ou à l'autre de ces traditions. Il serait également difficile de rattacher encore cette déclaration générale à une philosophie précise. Peutêtre avons-nous plutôt affaire à l'un de ces "lieux communs" mis en évidence par Jocelyne Dakhlia (1996, p. 52) :

«Un lieu commun est en effet, si l'on ose cette formulation intégrante, quelque chose de commun, qui rassemble en investissant des espaces qui sont, selon le cas, homogènes ou hétérogènes : il peut franchir les limites d'une culture ou y demeurer strictement circonscrit. Aussi dessine-t-il les contours de sa propre pertinence culturelle. »

14 Jusqu'à présent, je n'ai abordé que les points d'emblée négatifs par lesquels on définit l'Occident et les Occidentaux, ceux qui servent à la stigmatisation et à la typification. Mais les prêcheurs reconnaissent aussi des qualités à l'Occident et aux Occidentaux : le développement technique ou scientifique, le respect du travail, la valeur que l'on accorde au temps, la valorisation de l'être humain et le respect des promesses. Certes, ces qualités ont des liens entre elles, comme le remarque un prêcheur: "Ils sont développés scientifiquement et techniquement parce qu'ils respectent leur travail et parce que le temps, chez eux, à une valeur." Mais le développement scientifique est partagé en deux volets, les sciences sociales qui «sont subjectives et qui ont un but colonial et dont les méthodes ne peuvent pas s'adapter à nos sociétés, comme par 
exemple, la sociologie, l'anthropologie, la psychologie et la psychanalyse » et les sciences dures, si j'ose dire, comme la chimie, la physique, les mathématiques qui sont particulièrement valorisées. Selon les prêcheurs, elles ont d'ailleurs une origine arabe : «Tout ce que l'Occident possède en matière de civilisation et de progrès était à nous, nous en sommes les premiers inventeurs, les mathématiques et l'astrologie, par exemple, furent inventées par les Arabes. Les Occidentaux ont construit leur civilisation sur la base de la civilisation orientale. Mais malheureusement, ils ont pris le côté matériel et ont laissé le côté spirituel. Tout ce que l'on souhaite, c'est reprendre les choses qui rendent l'homme heureux, et cela après avoir fait une sélection afin de ne pas sortir de ce que recommande notre religion, l'islam ».

Ainsi les prêcheurs veulent-ils moraliser la science, c'est-à-dire l'insérer dans le cadre moral de l'islam, autrement dit, la rendre spécifique aux sociétés musulmanes : «On ne peut pas reprendre la science telle qu'elle existe chez eux (les Occidentaux), disent-ils, parce qu'ils suivent la méthode scientifique seule alors que nous, nous suivons la méthode religieuse qui est aussi scientifique. » Ce raisonnement peut paraître logique à partir du moment où l'on est d'accord sur le fait que tout est dans l'islam ou que l'islam traite de tout et qu'il n'a négligé aucun aspect de la vie. Mais il est remarquable que les prêcheurs ne parlent jamais de la manière dont on doit soumettre la science à la religion. Cela évoque assez directement le débat égyptien sur l'application de la sharî́a. On dit, en effet, que pour remédier à tous les maux de la société, il faut appliquer la sharî'a, ou encore que "nous sommes dans une situation critique parce qu'on n'applique pas la sharî'a». Mais comment appliquer cette référence qui n'a pas de contenu pratique ainsi que l'a bien montré Baudouin Dupret (1995) ? Le débat sur la moralisation de la science n'est pas né aujourd'hui mais existe depuis le mouvement réformiste :

« 'Abduh cherche à démontrer que l'Islam contient en lui-même les potentialités de cette religion rationnelle, cette science sociale et ce code moral qui pourraient servir de base à la vie moderne; et aussi à créer l'élite qui devrait le garder et l'interpréter, des oulémas d'un nouveau type qui peuvent formuler et enseigner le vrai Islam et fournir ainsi la base d'une société stable et progressiste, un "groupe médian" entre les forces traditionnelles et révolutionnaires qu'a montrées Comte et qu'on discerne si facilement dans la société islamique moderne.» (Hourani, 1991, p. 145)

16 En fait, moraliser la science, c'est trier et sélectionner, et cela est établi depuis le mouvement réformiste et Muhammad 'Abduh.

Or, cet Occident qui « veut continuer à dominer l'Orient » ne lui restitue pas la science mais lui donne, au contraire, ce qui relève de l'immoralité : «On ne refuse pas les bonnes choses qui peuvent venir de chez eux, la bonne parole je la prends et je l'applique, même si elle est dite par un prêtre, mais eux, ils nous exportent le mal et gardent le bien. » Cette rhétorique signifie que l'Occident ne veut pas exporter les «bonnes choses » en Orient mais, en revanche, veut le dépouiller de ses qualités, c'està-dire de son éthique, laquelle est considérée comme un bien précieux qui peut remplacer ou compenser le manque industriel. Ainsi, l'une des qualités reconnues à l'Occident se retourne-t-elle en défaut et, bien que ce dernier soit plus avancé dans le domaine industriel, il apparait, à l'inverse du monde musulman, comme un monde « inférieur » parce qu'il n'est pas moral.

J'ai dit que les prêcheurs reconnaissaient aussi à l'Occident et aux Occidentaux le respect du travail, la valeur donnée à l'être humain, la bonne organisation, la justice et 
le respect des promesses. Ici, on admet que l'Occident a une morale mais une morale d'une autre nature, car elle n'est pas religieuse. En effet, la morale de l'Occidental n'est pas religieuse dans la mesure où il ne respecte pas « ce qui lui reste de sa religion ». Elle est innée chez l'Occidental mais religieuse chez l'Oriental. Selon les prêcheurs, ces qualités de l'Occident sont présentes dans le Coran et les croyants sont incités à les faire leurs, mais elles ne sont pas présentes dans la réalité. Les prêcheurs souhaitent qu'elles soient répandues dans leur société et, dans ce cas précis, sans faire référence à l'islam s'il le faut. L'un d'eux dit d'ailleurs : «Si je parle de l'Occident dans mes prêches, je dirais aux musulmans : respectez votre temps, votre travail et votre parole. Ce n'est pas pour imiter les Occidentaux mais pour suivre ce qu'a dit notre Prophète, et si la parole du Prophète vous déplaît suivez en cela votre ennemi, les Occidentaux ont beaucoup de choses, prenez le bien et laissez le mal. »

\section{Les prêcheurs coptes et l'Occident}

On l'a vu, pour le prêcheur musulman l'altérité vis-à-vis de l'Occident est basée sur des questions qui relèvent de la religion et de la morale islamiques. Les prêtres coptes, eux - parce qu'ils sont chrétiens - se sentent-ils plus proches des Occidentaux, pensent-ils qu'ils partagent avec eux une même morale? Lorsque je dis prêcheurs coptes, je ne désigne que les prêtres de l'Église copte orthodoxe, ceux qui travaillent, dans l'optique définie par le pape Chenouda III, à une réactualisation du cadre de socialisation et d'identification tant public que privé constitué par l'Église copte (EI-Khawaga, 1992).

Les prêtres de l'Église copte parlent des Occidentaux d'une manière presque identique à celle des prêcheurs musulmans. Ils les qualifient d'immoraux, ils évoquent leur "déviance sexuelle », leur mauvaise utilisation d'une "liberté sans limites » ainsi que leur conception du mariage qui n'est pas celle des coptes :

«Le mariage chez nous, c'est pour la vie, c'est très sérieux. Eux, ils se marient sans réfléchir, le mariage chez eux est conçu comme une expérience, ils se disent : on se marie, si ça marche on reste ensemble; si ça ne marche pas, on divorce. La famille n'a pas d'importance chez eux et c'est pour cela que leurs enfants ont beaucoup de problèmes.»

21 Ils définissent les Occidentaux comme des êtres éloignés d'eux, l'éloignement ne voulant pas dire distance géographique mais distance culturelle. Ils n'ont pas les mêmes références éthiques ou, au moins, ils n'ont pas les mêmes attitudes morales. Pour les prêcheurs coptes, l'altérité se situe entre l'Occident et l'Orient, c'est-à-dire, entre les Égyptiens, qu'ils soient chrétiens ou musulmans, et les Occidentaux. La seule différence entre les prêcheurs musulmans et les prêtres coptes tient au fait que ces derniers ne se référent pas à un cadre religieux mais à un cadre "ethnique ». Ils se sentent plus proches des Égyptiens musulmans parce qu'ils sont, tout comme eux, des Orientaux. De ce point de vue, l'appartenance "ethnique» est plus importante que l'appartenance religieuse.

De leur côté, les prêcheurs musulmans, qui ont jusqu'à présent tenu un discours d'altérité basé sur la différence religieuse, partagent-ils cette conception de l'appartenance ? Considèrent-ils les coptes comme des êtres également immoraux du fait de leur christianisme ou adoptent-ils, à l'instar des prêcheurs coptes, une conception « ethnique » de la différence morale? 
Ces, lorsqu'on interroge les musulmans sur ce qui les sépare des coptes, la réponse tourne invariablement autour d'une certaine immoralité dont ces derniers seraient porteurs. Jean-Noël Ferrié (à paraître) qui a interrogé des étudiants musulmans de l'université d'Al-Azhar sur les facteurs de démarcation entre musulmans et coptes nous montre que, selon les critères de ces étudiants,

«ils sont différenciés par a) la religion, b) l'origine et c) les mœurs[...]. La distinction concernant les mœurs rétablit l'idée de souillure dans le discours de la démarcation identitaire, sans impliquer la définition d'une longue série d'interdits. Il suffit de dire que les femmes coptes sont impudiques dans leur mise pour établir une démarcation d'autant plus nette qu'elle s'affirme comme actuelle et visible dans la vie quotidienne ».

Ici, les coptes sont stigmatisés par leur religion et par le manque de pudeur de leurs femmes. Mais on peut toutefois remarquer quelques différences qui les distinguent des Occidentaux. Le manque de pudeur n'est pas exactement la « sexualité déviante » dont est taxé l'Occidental mais porte seulement sur la manière dont s'habillent les femmes (" elles ne sont pas voilées»). En ce qui concerne la religion, le copte est chrétien; certes, il a un texte (les «Écritures saintes ») falsifié mais il croit à ce texte, il le suit et il croit en Dieu, ce qui n'est pas le cas de l'Occidental qui se réfère aussi à un texte falsifié mais qui ne le suit même pas. Lorsqu'on est dans un débat endogène, c'est-à-dire, lorsqu'on parle des coptes et des musulmans égyptiens entre Égyptiens, certains musulmans peuvent stigmatiser le copte sur une base religieuse et éthique mais, lorsqu'un élément exogène intervient, on tient un tout autre discours, celui d'une commune appartenance « ethnique ». Autrement dit, lorsqu'on demande aux prêcheurs musulmans: "Quelles sont les différences entre un musulman égyptien, un copte égyptien et un Occidental? ", ils situent la différence uniquement entre Égyptiens et Occidentaux. Le copte a une éthique parce qu'on le compare à un Occidental. Face à un étranger lointain, le copte devient donc un Oriental qui possède la même éthique que les musulmans. Dans ce cas, l'appartenance locale devient plus pertinente que l'appartenance religieuse. Les prêcheurs musulmans font ainsi, dans certaines circonstances, abstraction de l'élément fondamental de leur identité revendiquée qui est l'islam, et utilisent un autre élément qui le remplace et qui devient alors aussi fondamental : l'appartenance à l'Orient. L'Orient ici, est réduit à l'Égypte et à son histoire millénaire qui explique son "authenticité » ou, comme disent à la fois les prêtres de l'église copte orthodoxe et les prêcheurs musulmans :

«Nous avons des origines lointaines communes. Nos origines sont les plus anciennes, elles datent des Pharaons. Nous avons toujours vécu dans l'authenticité et nous sommes des gens authentiques, nos valeurs communes sont aussi authentiques que les pyramides, nos filles et nos garçons, qu'ils soient chrétiens ou musulmans, respectent nos traditions, ils ne font pas n'importe quoi, ils n'ont pas de relations sexuelles en dehors du mariage. »

C'est l'authenticité qui produit les valeurs communes. Le terme authenticité (assâla) renvoie à un usage de l'idéologie identitaire, il signifie que telle personne ou tel groupe est conforme à la typification que l'on donne du groupe et que le groupe - ou ses représentants - revendique pour lui-même.

Certes, on trouvera des jugements de valeur opposant les Égyptiens coptes et musulmans. On ne peut pas davantage nier que des musulmans puissent considérer les chrétiens comme des personnes souillées, mais cela reste, pour eux, un problème égypto-égyptien, quelque chose d'interne qu'il importe de cacher à tout étranger. Ce 
qu'ils veulent montrer, c'est l'unité, c'est la cohabitation qui se déroule dans le calme et l'entente la plus parfaite. Toute personne qui provoque un incident pouvant exprimer une certaine inimitié vis-à-vis des coptes est traitée d'ennemi et de traitre, qui essaye de détruire cette unité nationale si chère aux Égyptiens. Une récente agression contre un monastère en Haute-Égypte a entraîné tout un mouvement en faveur de cette unité entre coptes et musulmans; tous les partis politiques, et même ceux qui sont perçus comme proches des « islamistes » ont dénoncé cette agression, les ministres, dont celui des Waqfs, et le cheikh d'al-Azhar allant jusqu'à présenter leurs condoléances aux familles des victimes, et déclarant que celui qui s'en prend aux coptes n'a qu'un seul objectif: détruire l'unité nationale et, de ce fait, s'attaquer à l'Égypte. Ce genre d'indignation fut aussi provoqué par le colloque que Sa'ad al-Dîn Ibrâhîm voulut organiser en 1994, en Égypte, et qu'il a fini par organiser à Chypre, sur les «minorités dans le monde arabe ». Le projet de ce colloque fut considéré comme un affront à la cohésion nationale. Le célèbre écrivain Hasanayn Haykal qui a déclenché une guerre contre ce colloque refusait d'inclure les coptes dans la catégorie des minorités, affirmant d'emblée que « coptes et musulmans appartiennent depuis toujours au même tissu national et civilisationnel ». Il est même allé jusqu'à soulever publiquement la question des «financements étrangers des colloques et de la recherche en sciences sociales ", ranimant ainsi le thème ô combien mobilisateur de l'affect nationaliste, du « complot externe » instrumentalisant les «problèmes des minorités » pour ébranler la cohésion nationale des sociétés arabes en général et de la société égyptienne en particulier (El-Khawaga, 1994).

$\mathrm{Au}$ total, on constate l'existence d'un consensus entre coptes et musulmans autour de l'instauration d'une différence globale entre Occidentaux et Orientaux. Cette distinction, on vient de le voir, a une utilité interne ; elle fonde une identité partagée : « Nous, les Égyptiens, nous sommes moraux, nous sommes authentiques, quelle que soit notre religion. »Ce consensus, bien sûr, n'est pas stable, parce que les prêcheurs musulmans peuvent toujours recourir à l'immoralité pour distinguer les musulmans des coptes. Mais cela montre principalement que les typifications fondées sur la moralité n'ont pas d'attributaires liés. En fait, on se contente de projeter sur des groupes divers le négatif des qualités que l'on se reconnaît.

\section{BIBLIOGRAPHIE}

DAKHLIA J., 1996, « La question des lieux communs. Des modèles de souveraineté dans l'Islam méditerranéen », dans LEPETIT B., Les formes de l'expérience, Paris, Albin-Michel.

DUPRET B., 1995, «La shari'a comme référent législatif : du droit positif à l'anthropologie du droit ", Revue interdisciplinaire d'études juridiques.

FERRIÉ J.-N., à paraître, « Qu'est-ce qu'un copte selon les musulmans ? Construction de la frontière et typification de soi ", dans DÉCOBERT C, Dynamiques identitaires en Égypte.

HOURANI A., 1991, La Pensée arabe et l'Occident, Paris, Naufal. 
IRETON F., à paraître, « Les quatre relations d'incertitude d'un construit identitaire collectif à référence territoriale : l'exemple des Sa'îdî», dans DÉCOBERT C, Dynamiques identitaires en Égypte.

EL-KHAWAGA D.

- 1994, « Le débat sur les coptes : le dit et le non-dit », Égypte-Monde arabe, nº 20.

- 1992, «L'affirmation d'une identité chrétienne copte. Saisir un processus en cours », dans DECOBERT C, Itinéraires d'Égypte. Mélanges Maurice Martin, Le Caire, IFAO.

LAROUI A., 1986, Islam et Modernité, Paris, La Découverte.

LÉVI-STRAUSS C, 1973, Anthropologie structurale II, Paris, Pion.

SCHUTZ A., 1987, Le Chercheur et le Quotidien, Paris, Méridiens-Klincksieck.

INDEX

Mots-clés : identité, Occident, Coptes

\section{AUTEUR}

SAADIA RADI

Cedej 